\title{
Deriving a useful packed cell volume estimate from haemoglobin analysis
}

\author{
G O’Connor, A M Molloy, L Daly, J M Scott
}

\begin{abstract}
A method is described whereby the packed cell volume (PCV) of a blood sample can be estimated from the haemoglobin concentration. The method defines the relation existing between haemoglobin concentration and the PCV of blood samples and uses the mathematical formula thus obtained to estimate an unknown PCV value for a sample by extrapolation from the known haemoglobin value of the same sample.

The method could be used in large scale field studies where haematological services are not available and where an evaluation of red cell status of vitamins such as folate is required.
\end{abstract}

( Clin Pathol 1994;47:78-79)

Red cell folate content is considered to be the best index of tissue folates. ${ }^{1}$ This is derived by combining a whole blood folate measurement with the packed cell volume (PCV) of the sample, measured by haematocrit or electronic counter. Our laboratory is involved in nutritional field studies involving large numbers of out patients for whom backup haematological services are not available. It has thus become necessary to obtain an estimate of the PCV indirectly to provide a red cell folate result.

It has long been recognised that there is a close relation between an individual's PCV and haemoglobin concentration. ${ }^{2}$

Pathology

Laboratories, $\mathbf{S t}$

James's Hospital,

Dublin 8, Ireland

G O'Connor

Department of

Clinical Medicine,

Trinity College,

Dublin 8, Ireland

A M Molloy

Department of

Biochemistry, Trinity

College, Dublin

J M Scott

Department of Public

Health Medicine and

Epidemiology,

University College

Dublin

L Daly

Correspondence to:

Dr A M Molloy

Accepted for publication 5 July 1993

\section{Methods}

Results were collected from randomly selected blood samples processed for routine haematological examination in this department. All had been analysed using a Coulter $\mathrm{S}+$ IV model, calibrated with $4 \mathrm{C}+$. The instrument was registered as part of an external quality assessment scheme during the study. The data were divided into categories as follows: $\mathrm{MCV}$ of $<80 \mathrm{fl} ; 80 \mathrm{fl} \leqslant \mathrm{MCV} \leqslant$ $94 \mathrm{fl}$; and $\mathrm{MCV}$ of $>94 \mathrm{fl}$, to reflect the approximate cutoffs for low, normal, and high MCV values. Microcytic anaemias occur below the $80 \mathrm{fl}$ cutoff and macrocytic anaemias above the $94 \mathrm{fl}$ level, so the possibility of altered correlations within these cate- gories was taken into account. The range of haemoglobins (and number of samples) in the low, normal, and high MCV groups were, respectively, 72-176 ( $\mathrm{n}=104) ; \quad 72-182$ ( $\mathrm{n}=57)$; and $63-167 \mathrm{~g} / \mathrm{l}(\mathrm{n}=74)$. No other criteria were used to include or eliminate samples. Regression analysis was carried out for haemoglobin concentration against PCV. To avoid the possibility of bias, separate regressions were performed in each $\mathrm{MCV}$
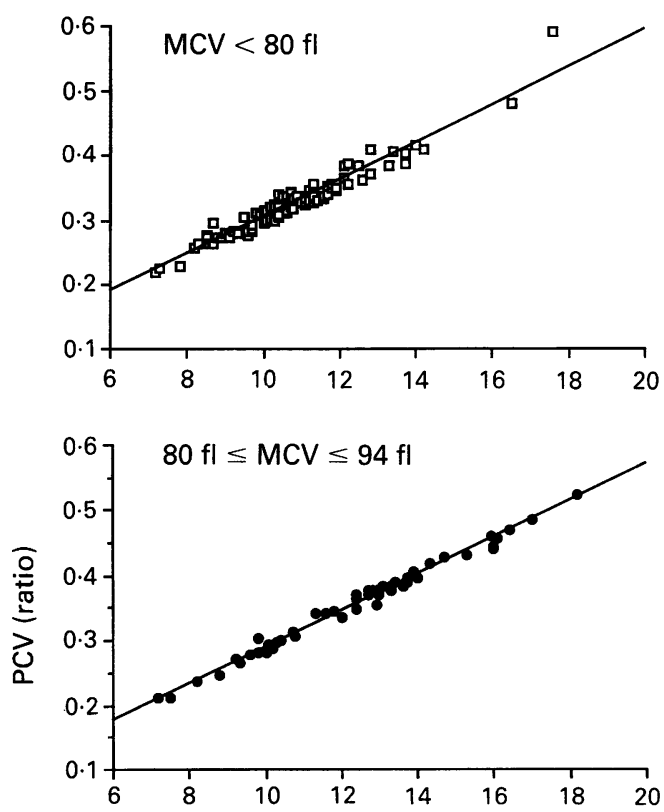

blood samples stratified by $M C V$ as follows:

(1) $M C V<80 f(n=104) ; P C V=0.029+0.015$; $r=0.97$; residual $S D=0.013$.

(2) $80 \mathrm{fl} \leqslant M C V \leqslant 94 \mathrm{fl}(n=57) ; P C V=0.028+$

$0.008 ; r=0.99 ;$ residual $S D=0.008$

(3) $M C V>94 f(n=74) ; P C V=0.030-0.015$;

$r=0.98$; residual $S D=0.014$ 
group rather than constraining the estimates by fitting parallel regressions.

\section{Results and discussion}

The results are given in the figure. Within each MCV group there was a strong linear correlation between haemoglobin concentration and PCV. This was only marginally affected by $\mathrm{MCV}$, and maximum discrepancies occur at very low or very high haemoglobin concentrations. For example, a haemoglobin concentration of $60 \mathrm{~g} / \mathrm{l}$ would give a PCV of 0.176 against the normal MCV curve, $0 \cdot 189$ against the low MCV curve, and $0 \cdot 165$ against the high MCV curve. There was no substantial difference in the regressions of the low MCV curve when analysed without the high value outlier $(\mathrm{PCV}=0.027$ haemoglobin +0.033 ).

To validate the technique, blood count data were collected from 25 subjects with haemoglobin values ranging from $43 \mathrm{~g} / 1$ to $160 \mathrm{~g} / 1$ and $\mathrm{MCV}$ values ranging from $67 \mathrm{fl}$ to $140 \mathrm{fl}$. The PCV was estimated from the derived equation for normal $\mathrm{MCV}$ and the results were compared with PCV results obtained using the Coulter counter. The correlation coefficient was 0.99 (data not shown). Agreement between the methods was assessed using the technique described by Bland and Altman. ${ }^{3}$
On average, the new haemoglobin based method of determining PCV gave an estimate of 0.019 ( $95 \%$ confidence interval 0.013 to $0.025)$ lower than the direct Coulter method. This represents a downward bias of $4.8 \%$ on a PCV of 0.40 . The scatter of the discrepancies suggests that in $95 \%$ of people the new method will give values between 0.049 lower and 0.011 higher than the Coulter result. In carrying out surveys of large populations this level of error in red cell folate is within acceptable limits.

As a final comment, we have also found that where a value for haemoglobin is not available, the standard Drabkin's method of analysis gives excellent results when carried out on dilutions of whole blood into $1 \%$ ascorbic acid. Quality control standards should also be diluted into ascorbate and a drop $(10 \mu \mathrm{l})$ of ammonia solution added to all tubes to remove turbidity, as described elsewhere. $^{4}$

Chanarin I. The megaloblastic anaemias. 2nd edn. Oxford: Blackwell Scientific, 1979:187-97.

2 Dacie JV, Lewis SM. Practical haematology. 7th edn. London: Churchill Livingstone, 1990:11-5.

3 Bland JM, Altman DG. Statistical methods for assessing agreement between two methods of clinical measurement. Lancet 1986;i:307-10.

4 Tentori L Salvati AM. Hemoglobinometry in human blood. Methods Enzymol 1981;76:707-15. 